\title{
Service Visibility Across Lines Of Business
}

Karen L. Harper, Bank of America, USA

\begin{abstract}
This paper introduces the use of service science to maximize profitability by providing service transparency across lines of business in a financial operating environment. The concept of a live collector that runs in the cloud is presented. The collector gives information visibility, context mediation, and presentation facilities on a dynamic basis by employing the principles of cloud computing. The subject of cloud computing is introduced in the paper.
\end{abstract}

Keywords: Cloud Computing; Service Visibility; Context Mediation; Live Collector

\section{INTRODUCTION}

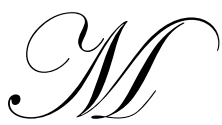

uch of modern business is dependent upon information that is locked in on-premises information systems. Clearly, the price for maintaining and accessing this information is high and incorporates infrastructure, hardware, software, development, and professional services including in-house costs of utilization and deployment. Information systems of this sort have evolved such that the aforementioned information can be encapsulated, exposed as components, and accessible as services over the Internet. In many cases, the components are usable in systems development for the development of other on-premises software applications. [6] The concept is well established and known as Service-Oriented Architecture (SOA). [2, 4] The drawback of most, but certainly not all SOA applications is that the up-front costs are high, time consuming, and yield only a marginal contribution to business agility. Many business operations require that information from several on-premises system be visible for consistent and effective decision-making. The provisioning of information from several systems across differing lines of business is the subject of this paper. [1]

\section{CLOUD COMPUTING CONCEPTS}

Cloud computing is a collection of technologies for accessing computer facilities via the Internet. From a user perspective, ubiquitous access is provided to software and information through the use of a web browser. The adjective "cloud" reflects the diagrammatic use of a cloud as a metaphor for the Internet and suggests that the same services are available in several forms, such as conventional web access, mobile platforms, and mesh computing. [5, $7,8,9,10]$

From a provider perspective, cloud computing refers to software deployed as a hosted service and accessed over the Internet. There are two aspects to service provisioning: infrastructure services and application services. Both use a cloud platform, which is essentially an operating system that runs in the cloud. [3, 10] Infrastructure services include authorization/authentication/security facilities, integration between infrastructure and application services, and online storage facilities. Application services refer to ordinary business services that expose "functional" services as SOA components. Cloud platforms are a lot like enterprise-level platforms, except that they are designed to scale up to Internet-level operations supporting millions of clients.

Cloud computing clients are grouped into three categories: traditional business users, independent software vendors (ISVs), and consumers. Traditional business users run their applications in the cloud to avoid up-front deployment costs and to minimize development and deployment time. ISVs develop multi-tenant applications that can address a wider marketplace for services and provide economy of scale for the client. Cloud consumer services are typically free to the client, as well as being accessible from any location via the Internet, and yield advertisingsupported revenue for the provider. Consumer services have a near-zero marginal cost of distribution to clients. 


\section{APPLICATION ARCHITECTURE}

For the purposes of this paper, two forms of cloud application architecture are established: the multi-tenant model and the visibility model. The multi-tenant model runs on a cloud platform and uses a common data model, for all tenants. The model provides economy of scale for clients based on long tail monetization. [6] This model, popular with ISVs, probably will use the virtualization feature of cloud operating systems to achieve multi-tenancy, and requires that all clients run the same basic business application. [3]

The visibility model reflects proprietary software that runs on a cloud platform. It uses a single tenant model and demands a separate data model. Each tenant may have several users, each associated with the same client. Internet access is required for on-premises and other cloud applications.

\section{SYSTEM DESIGN}

Achieving service visibility across lines of business requires a software application that is hosted on a cloud platform and adopts the visibility model. We are going to call this application the live collector that creates an operational scenario on a dynamic basis. The collector is script driven and creates an instance for each user. It is important that the application runs as a client, where the client can sustain several users. An application instance is synthesized for each user. Figure 1 gives a structural and process design diagram of the live collector with functional cub-components, as required. The main components are the script-driven instance generator, the accesser to on-premises data objects, the synthesizer that derives a composite of the application domain, the presenter that derives the requisite dashboard, and a storage manager for handling the data-centric aspects of cloud computing. The systems environment is conceptualized in Figure 2.

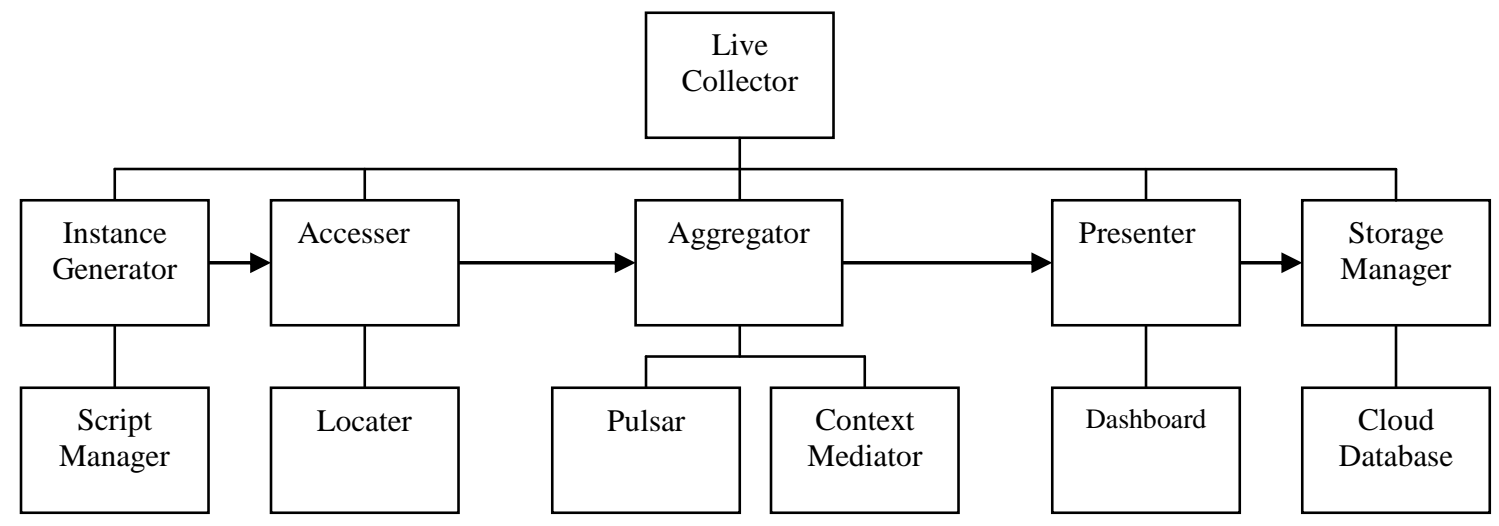

Figure1: Design of the Live Collector

\section{Instance Generator}

The live collector is composed of software components that can be assembled dynamically to instantiate a specific application instance. The instance generator is a script-driven functionality that generates an operational instance. This operational instance, when executed, will access components from on-premises information systems with the accesser component and then synthesize a composite data structure, relying on the context mediator when needed. The composite data structure will feed the presenter component that generates and updates the dashboard. The synthesizer will employ a pulsar module that can dynamically refresh the display from on-premises components. The storage manager, also script driven, will store operational information in a cloud database. Since the instance runs in the cloud, it can be accessed from any location and with fixed and mobile facilities. 


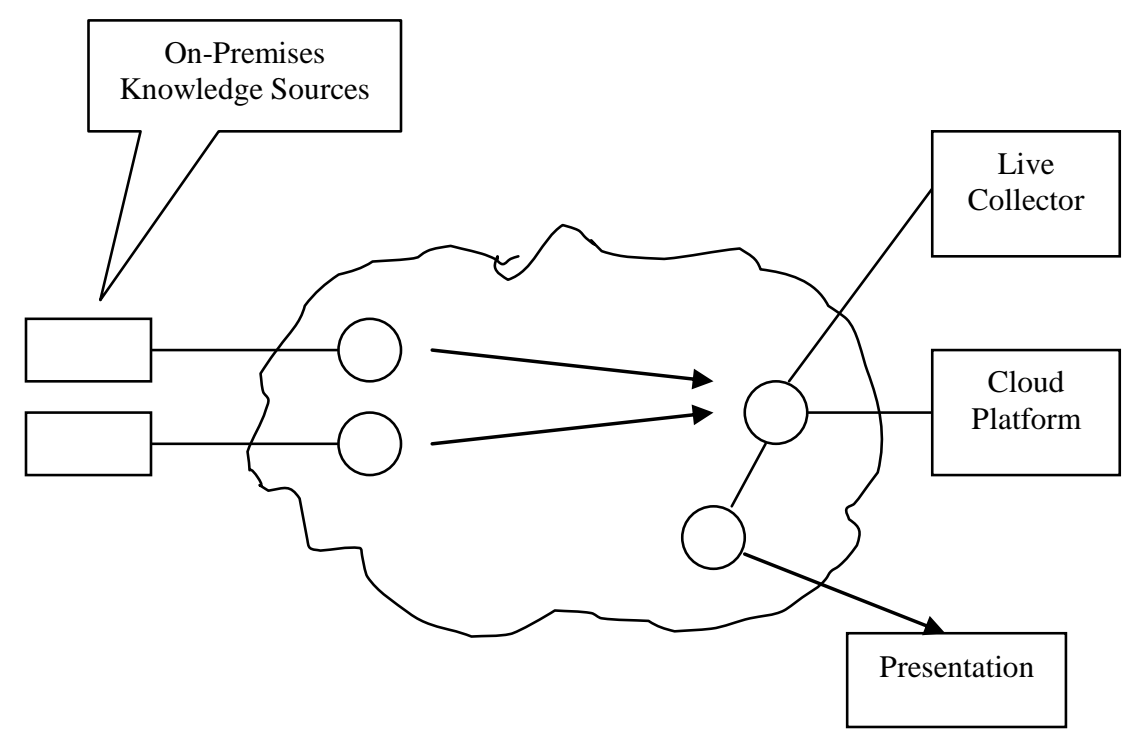

Figure 2: Conceptualization of the Live Collector

\section{Accesser}

The primary function of the accesser component is to locate needed information on on-premises information systems. To achieve data location, the accesser will employ Web Services technology, which includes a registry of system components. [2] The locater looks up needed components in the registry and returns their Internet-accessible reference locations. The accesser can return that information to the synthesizer for subsequent composition and display,

\section{Synthesizer}

The synthesizer is a software component that resides in the live collector with the sole objective of combining information as prescribed in the script. The synthesizer is conceptualized as a rule-based software component that would necessarily employ the services of a "context mediator" to resolve semantic differences in otherwise incompatible entities, as described in the following quotation. "Context mediation is a field of research that is concerned with the interchange of information across different environments, which provides a vehicle to bridge semantic gaps among disparate entities." [1] To insure the timeliness of information, the synthesizer should exercise a pulser component that would refresh information on a periodic basis.

\section{Presenter}

The presenter is an open-ended display component - often regarded as a "dashboard" - designed to match informational requirements with display technology. Presenter output is expected to be archived for subsequent reference and to protect against repudiation.

\section{Cloud Storage Manager}

The cloud storage manager is a front-end to a cloud database component designed to handle cases of multitenant data structures with multiple users. A key issue with cloud storage management is "Who owns the data?" It is generally felt that ownership considerations can be resolved with effective service-level agreements.

\section{Workflow}

As implied in Figure 1, the workflow for service visibility is the following: instance generation, access, synthesis, presentation, and storage. Since instance execution is script driven, the flow can be adjusted on a dynamic basis, perhaps with the use of intelligent agents. [11] 


\section{SERVICE VISIBILITY CASE STUDY}

Transparency is a favorite buzzword on Capitol Hill given today's economy and this provides corporate America a vehicle to address those needs both internally and externally. Internally, decision making can be done with a full view of other business relationships using the dashboard view. Externally, we provide shareholders and auditors, alike a vehicle to gather data without the time consuming drilling into numerous arms of a business as employed in the past.

\section{BUSINESS PERSPECTIVE}

Practical applications to leverage this technology are numerous, extending beyond the obvious infrastructure savings an entity can realize. Infrastructure cost reduction attributable to external housing of hardware can be realized immediately. In addition, infrastructure and occupancy costs can be decreased while realizing gains in employee work life balance and satisfaction by using the virtual environment to reconfigure workforce geography. Geographical reconfiguration is not limited to where staff is housed allowing for expansion of work from home opportunities reducing infrastructure and occupancy but allows us to tap into the talent bank in an international way without onerous relocation costs which limited an entity's talent bank in years past. This methodology helps corporate America address country wide initiatives in the workplace to include migration of work force to home based work solutions to minimize real estate/occupancy costs; minimize use of natural resources and maximize work-life balance and tap into resources internationally but uses extend further.

The most exciting opportunity afforded by utilization of cloud computing allows us to address transparency needs of both multi-billion and million dollar entities operating multiple lines of products and/or services to enhance understanding of Customer CTQ's [Critical to Quality factors] and profitability by enhancing services and packaging of solutions. Cloud computing also addresses the Voice of the Customer [new Administration and shareholder] demands for transparency allowing for visibility across lines of business resulting in same for shareholder.

Cloud computing will allow for a line of business to access information in a dashboard view integrating information and evaluating scenarios to maximize packaging of customer solutions. This dashboard view would provide information for decision making on a given customer to enhance understanding of customer needs and allow for packaging of solutions to maximize satisfaction and profitability. Resource use is minimized through the use of the cloud where capacity on the desktop is not necessary. Risk is mitigated by allowing access based on levels of security and providing only public type information. This dashboard will not only serve as a tool on a daily basis but allow the business to monitor and analyze customer needs and modify as market trends dictate. (Figure 3)

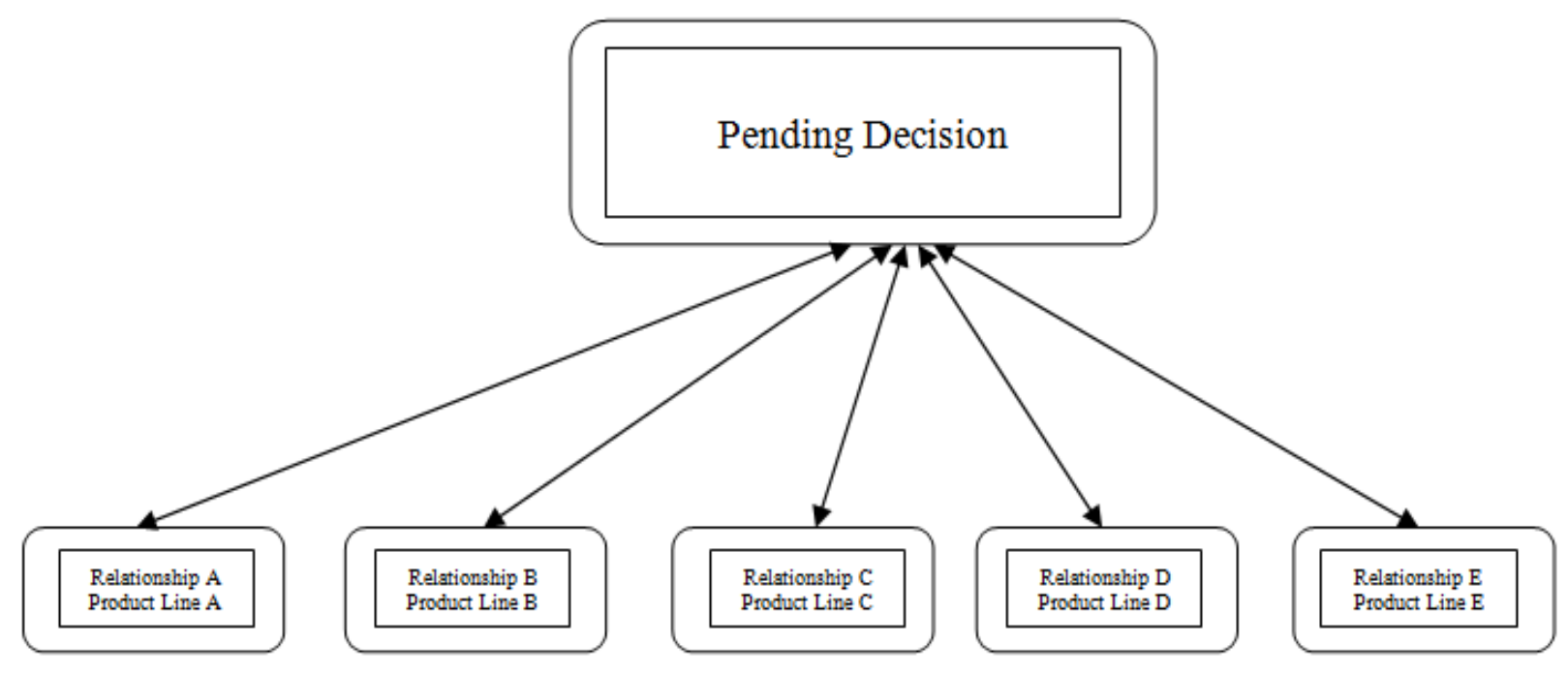

Figure 3: Dashboard View Creates A Decisioning Tree Which Allows Full Visibility To Other Relationships With No Limitations Created By Geography, Infrastructure, Or Software Requirements. 


\section{QUICK SUMMARY}

1. The basis for service visibility is service science, cloud computing, Web Services, and Service-Oriented Architecture.

2. Cloud computing is a collection of technologies for accessing computer facilities via the Internet.

3. The two aspects of cloud service provisioning are infrastructure services and application services.

4. Cloud computing clients are grouped into three categories: traditional business users, independent software vendors (ISVs), and consumers.

5. The two forms of cloud application architecture are the multi-tenant model and the visibility model.

6. A live collector is a software application used to achieve service visibility across lines of business.

7. The main components of a live collector are the instance generator, the accesser, the synthesizer, the presenter, and the storage manager.

8. Business applications can be designed to promote transparency employing a dashboard view that crosses all boundaries of geography; infrastructure and software allowing a business to use a decisioning tree with a view of all relationships within an organization.

\section{AUTHOR INFORMATION}

Karen L. Harper is a Senior Financial Professional with 20+ years of experience in Finance, Accounting and Project Management. Currently engaged as an Operations Manager with Bank of America - work experiences span from financial management to strategic decision making via evaluation of financial impacts of business decisions, and communication and presentation of complex financial problems and solutions to multiple levels of management. Innovative approaches to improve both the accuracy and positive impact of decision making has been the focus of her career. Ms Harper has also engaged as an Adjunct Accounting Professor at both Strayer University and Montreat College. E-mail: karen.1.harper@bankofamerica.com

\section{REFERENCES}

1. Buchner, A., Context Mediation among Knowledge Discovery Components, www.dissertation.com, (from the abstract), (2004).

2. $\quad$ Cerami, E., Web Services Essentials, Sebastopol, CA: O’Reilly Media, Inc., (2002).

3. Chappell, D., "A Short Introduction to Cloud Platforms," Microsoft Corporation, (August 2008).

4. $\quad$ Erl, T., SOA: Principles of Service Design, Upper Saddle River, NJ: Prentice-Hall Inc., (2008).

5. Katzan, H., "Cloud Computing, I-Service, and IT Service Provisioning," Journal of Service Science, Volume 1, Number 2, (2008).

6. Katzan, H., Foundations of Service Science: A Pragmatic Approach, New York: iUniverse, Inc., (2008).

7. Katzan, H. (2009). Cloud Computing Mesh Model for Decision Support Systems. Myrtle Beach SC: Southeastern INFORMS Conference.

8. Knorr, E and G. Gruman, "What cloud computing really means," InfoWorld, April 07, 2008, www.infoworld.com, (2008).

9. Martin, R and J. Hoover, "Guide to Cloud Computing," Information Week, June 21, (2008), www.informationweek.com, (2008).

10. Miller, M., Cloud Computing: Web-Based Applications That Change the Way You Work and Collaborate Online, Indianapolis: Que Publishing, (2008).

11. Russell, S. and P. Norvig, Artificial Intelligence: A Modern Approach (2ed), Upper Saddle River, NJ: Prentice-Hall Inc, (2003). 


\section{NOTES}

